\title{
Using Fine-Grained Infrared Positioning to Support the Surface-Based Activities of Mobile Users
}

\author{
Albert Krohn, Michael Beigl \\ Telecooperation Office \\ Unversität Karlsruhe
}

\author{
Mike Hazas, Hans-Werner Gellersen \\ Computing Department \\ Lancaster University
}

\author{
Albrecht Schmidt \\ Institut für Informatik \\ Ludwig-Maximilians-Universität München
}

\begin{abstract}
Knowledge of the fine-grained location and orientation of devices on a surface can be used to enhance the surface-based computing tasks of mobile users in the home and workplace. However, existing systems which provide surface-based positioning information are often not a practical solution for mobile users, since the systems all rely upon pre-installed and calibrated environmental infrastructure. In this paper, we present prototype positioning devices for surfaces which do not rely on such infrastructure. We show that inexpensive infrared transducers can be used to effectively sense relative location and orientation of surface devices. We evaluate the novel approach of using intensity of light pulses for fine-grained location measurements.
\end{abstract}

\section{Introduction}

In everyday living and working environments, people perform a large number of tasks on surfaces such as desks and tables. For mobile users, these surface-based tasks often heavily involve portable computing devices, ranging from laptops to mobile phones. During such tasks, the ability to configure wired and wireless connections between the devices can be crucial. For meetings and collaborative work, it is commonplace for multiple users to have several devices each, and to utilise some form of shared virtual workspace between the devices.

Such application scenarios can be greatly enhanced using knowledge of the fine-grained location and/or orientation of the devices on the surface. Current location systems which can supply surface-based positioning data are thus inappropriate, since they rely on costly and labour-intensive installations of transducers and equipment in the environment.
This paper presents a novel prototype system which allows users' mobile devices to perform peer-to-peer sensing on a surface and compute their locations and orientations in a $2 \mathrm{D}$ space. We first outline specific surface-based applications for mobile users, and propose infrared light as a fine-grained location sensing medium appropriate for their portable devices. We then detail our implementation and characterisation of the prototype system. Our findings show that such a system supports the positioning requirements of mobile users' surface-based tasks.

\section{Related Work and Motivation}

This section first outlines applications which require the location and orientation of user devices placed upon surfaces, such as desks and meeting tables. It is then demonstrated that current location technologies are inappropriate for mobile users and their portable devices. The choice of infrared as a fine-grained, 2D location sensing technology for these devices is then justified.

\subsection{Surface-based activities of mobile users}

The relative positions and orientations of devices on surfaces can be used to enable a variety of applications highly relevant to mobile users. As proposed by Scott et al., actions such as PDA-to-laptop synchronisation and digital camera image downloading can be automatically initiated when the two devices concerned are placed in close proximity, and the source device is oriented to face the destination device [1]. The authors also suggest that positioning information is used to configure a temporary interface export from a small device to a larger one. For example, a PDA might be placed next to a laptop to allow a person to use the laptop's keyboard and touchpad to manipulate documents stored on the PDA. 
Often, many devices are placed on a single working surface, including keyboards, monitors, laptops, PDAs, mobile phones, and digital cameras. Their relative positioning information can be used to create GUI representations of the layout of the devices on the table. These allow the user to easily specify source and destination of data by performing drag-and-drop actions between devices across the surface (hyperdragging) [2].

Configuring connectivity between devices is also extremely important for mobile users who gather for teamoriented tasks, as shown previously [3]. Positioning information of the users' portable devices, such as laptops, mobile phones, and PDAs, can be used to facilitate easy transfer of data objects between team members, as well as automatic configuration of associations between devices. For example, using a laptop with a GUI representation of the devices on the table, one person can associate some data with a small, nearby device, making it a token for the data (similar in concept to the mediaBlocks presented by Ullmer et al. [4]). The token can then be handed to another group member, who can place it in close proximity to their own device to indicate that the system should transfer the data.

Alternatively, the token might represent a shared meeting resource, such as a projector. As the meeting progresses the token can be passed between participants, each of whom can display content on the projector by placing the token near and pointing it toward their own device. A URL or file specified by the user possessing the token could then be transferred temporarily to the PC driving the projector, which then displays the data object. (In this way, the token would provide some of the functionality of the MeetingMachine Remote Control, described by Barton et al. [3].) The projector token might also be placed in the centre of the meeting participants' table, and then its orientation used to indicate the device which should control the content displayed on the projector.

In modern devices, wireless communication such as Bluetooth or WiFi is often available. Achieving the above discussed applications is then only a matter of software. Most of the application scenarios described involve configuring connections and transfers between devices on the surface. Thus, the necessary positioning information provided to the applications need only be relative (referenced to the devices themselves) rather than absolute (referenced to the surface or the room). The positioning requirements of the applications discussed can be summarised as follows:

1. Location accuracy: between $10 \mathrm{~cm}$ and $20 \mathrm{~cm}$, a granularity similar in size to the smaller devices which might be used (mobile phones and PDAs)

2. Orientation accuracy: about $30^{\circ}$, allowing detection of when devices are facing one another
3. Update rate: once every few seconds, providing adequate response time for configuring connections

4. Number of devices: up to twenty for large meetings or mobile users with many devices on a single surface

\subsection{Existing location technologies appropriate for surfaces}

Certain indoor location systems for context-aware computing are able to provide fine-grained location and orientation information sufficient for applications on surfaces $[5,6]$. Systems have also been developed specifically for surface-based location sensing. These have used a number of techniques, including computer vision $[2,7,8]$, physical contact or weight $[9,10]$, optical mouse tracking [11], and short-range electromagnetic signals [12].

The indoor location systems mentioned above accomplish wide-area fine-grained tracking, while the majority of the surface-based systems have been designed for user interface scenarios which require centimetre resolution and many updates per second. As a result, many of these systems have a high cost due to their sensor density, specialised transducers, or installation and calibration effort. Moreover, all of these systems require static, pre-installed infrastructure. For mobile users who wish to easily connect their portable devices wherever they go, or hold spontaneous meetings enhanced by location-enabled, shared virtual workspaces, systems requiring augmentation of the environment are impractical.

\subsection{Enabling surface-based location sensing on devices}

In order to avoid reliance on pre-installed tracking infrastructure, users' devices must incorporate sensing technology. The inclusion of the sensing technology should not adversely affect the battery life, size, or cost of the mobile devices. Accordingly, the transducers chosen should have a low power consumption, small form factor, and low signal processing overhead.

Computer vision and ultrasonic sensing technologies have been shown to be successful for fine-grained location, and could be incorporated into user devices for surfacebased location. For example, low-resolution cameras have been incorporated into some mobile phones, PDAs, and laptops. However, providing $360^{\circ}$ coverage around a device would involve using many cameras or ultrasonic transducers, potentially raising the cost, size, and complexity of the devices to a prohibitive level.

Conversely, a wide selection of infrared sensing technologies are available which are less expensive, smaller, 
and have lower signal processing requirements than computer vision or ultrasound-based techniques. Previously, infrared has been used for location systems with coarse granularity, ranging from room-scale to metre-scale accuracy. Examples include the Active Badge [13], ParcTab [14], Locust Swarm [15], and a system for wearable computing [16]. These systems rely upon infrared signal visibility between a fixed unit in the environment and a mobile unit, such as a device or tag. If a signal is visible, then it is assumed that the mobile unit is in the same area as the fixed unit, allowing a location to be inferred.

The HiBall system, designed for augmented and virtual reality, uses infrared to accomplish fine-grained tracking [17]. User-worn devices consisting of lateral effect photodiode clusters are used to accurately detect the angle of arrival of signals from arrays of ceiling-mounted infrared LEDs with known positions, and the location and orientation of the user is tracked using Kalman filtering. The system has the capability for sub-centimetre and sub-degree tracking resolution, but the user-worn devices are large and contain specialised hardware, making them inappropriate for widespread incorporation into portable devices such as mobile phones or PDAs.

In contrast to previous infrared systems, we propose measuring the intensity of infrared signals between portable devices on surfaces. The aim of using infrared technology is to achieve fine-grained location and orientation sensing at little additional cost, size, or complexity to user devices, while still avoiding reliance on pre-installed infrastructure.

\section{Prototype Implementation}

Figure 1 shows the implementation which has sixteen infrared emitters, twelve infrared receivers and additional components including analog multiplexers and filters, power circuitry and a microprocessor. The twelve photodetectors each cover $30^{\circ}$ and the emitters $22^{\circ}$, therefore the angle measurement errors should be limited to $\pm 15^{\circ}$ or $\pm 11^{\circ}$ respectively. Additionally, the wireless platform particle computer ${ }^{1}$ was used to organize the distributed control, synchronization and exchange of measurements. It is important to note that these prototypes were designed to facilitate flexibility, in order to fully gauge the potential of infrared technology for fine-grained, surface-based positioning. The measurements are based on a series of short (each $250 \mu \mathrm{s}$ ) infrared pulses. The receivers include analogue filters and a 10bit $35 \mathrm{kHz}$ A/D Converter. A single pair-to pair measurement between two devices for any range up to $250 \mathrm{~cm}$ and $360^{\circ}$ coverage takes $600 \mathrm{~ms}$ in the current implementation. The emission and reception of infrared pulses is organized in a one-to-many style. Many objects can measure their in-

\footnotetext{
1 http://particle.teco.edu
}

coming signals while only one object emits pulses. This improves the scaling behavior when the number of objects increases.

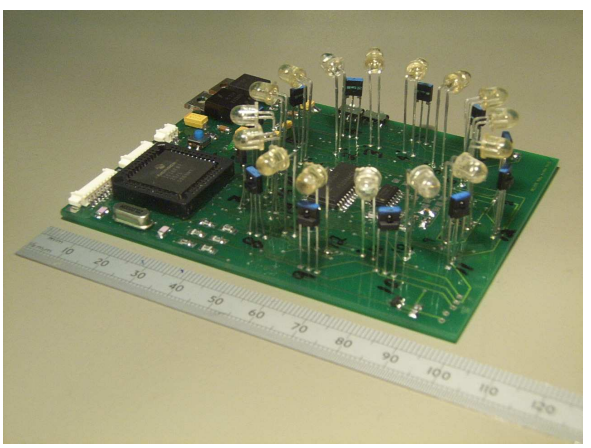

Figure 1. Infrared device prototype

\subsection{Location and orientation algorithms}

As different devices emit infrared pulses and receiving devices measure and broadcast their results, angles of emission/reception and inter-device ranges can be gathered for pairs of devices on the surface. Two methods for estimating the relative locations and orientations of the devices are presented here.

Map tracing. A collection of the distance and angle readings reported can be used to construct a virtual map, whose nodes represent devices and whose segments represent the distance and angle measurements between the devices. The map is then processed as follows:

1. Choose one device as the reference for the coordinate system.

2. Call a device with measurements towards another device as connected to it.

3. Use trigonometry and the distance/angle readings of devices connected to the reference device and place those devices on the map. Mark the already placed devices as "visited".

4. Choose a node which has not been visited, and calculate locations and orientations for any devices connected to it which has been placed already. Place the new node as well and mark it as "visited."

5. Repeat the previous step until the location and orientation for all devices in the map are calculated.

Thus, the algorithm traces the map to estimate the relative locations and orientations of the devices. The advantage of this method is that it is computationally lightweight; the 
map tracing algorithm can be executed in real time, even using a modest microcontroller such as the PIC employed in the prototype.

Non-linear regression. Alternatively, the angle and range measurements can be used to arrive at a solution for the relative locations and orientations of the devices using a system of equations.

More specifically, taking device $i$ and $j$, with locations $\left(x_{i}, y_{i}\right)$ and $\left(x_{j}, y_{j}\right)$, the euclidean distance $d_{i j}$ between them is:

$$
d_{i j}=\sqrt{\left(x_{i}-x_{j}\right)^{2}+\left(y_{i}-y_{j}\right)^{2}} .
$$

Also, the emission or reception angle $\psi_{i j}$ at device $i$ with respect to device $j$ can be related to $\theta_{i}$, the orientation of device $i$ referenced to the arbitrary coordinate system being used by the relative positioning system

$$
\psi_{i j}=\phi_{i j}\left(x_{i}, y_{i}, x_{j}, y_{j}\right)-\theta_{i},
$$

where $\phi_{i j}$ is the angle of the vector drawn from device $i$ to device $j$, as shown in figure 2 . As indicated in the equation, $\phi_{i j}$ is a function of the locations of the devices $i$ and $j$, and can be calculated using trigonometry.

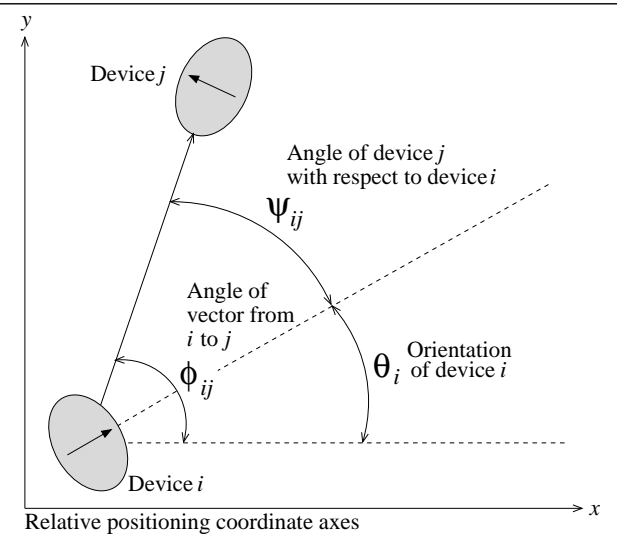

Figure 2. Device angle relationships

Since the devices in the system report measurements of the distances $d_{i j}$ and the angles $\psi_{i j}$, a non-linear regression algorithm can be applied to arrive at estimates for the devices' relative locations and orientations on the surface. The regression process converges toward location and orientation estimates which minimise the sum of the squares of the residuals (i.e. the difference between devices' measured distances and angles, and the distances and angles as calculated using equations 1 and 2). The solution can be further refined by using Studentized residuals to identify distances and angles which are likely to have large errors, and repeating the regression with those measurements discarded.
These techniques have previously been shown to work well in positioning systems [18].

\section{Experiment and Discussion}

This section presents a characterisation of the distance and angle measurements from the prototype devices, and the resulting location and orientation estimates. The practical utility of such a system for mobile users is then evaluated. For the test setting, four prototype devices were placed in one hundred different configurations on a $160 \times 200 \mathrm{~cm}$ surface indoors. For each configuration, the locations and orientations of the devices were arbitrarily chosen, with the aim of achieving an even distribution of locations and orientations over all the test configurations. Ambient light during the experiments came from overhead fluorescent lights and indirect sunlight.

\subsection{Distance and angle accuracy}

Figure 3 shows the error characteristic of the distance and angle measurements taken by the devices. Ninety percent of the distance measurements were within $18 \mathrm{~cm}$. Although the chosen photodetectors each covers a fairly broad angular range, the beam intensity of the emitters used in the prototype is greatly attenuated when the emitter is turned by ten degrees or more. When a receiving device does not lie within the main lobe of sensitivity of any of the transmitting device's emitters, measurements become less accurate. A simple software model to compensate for this was in place on the devices.

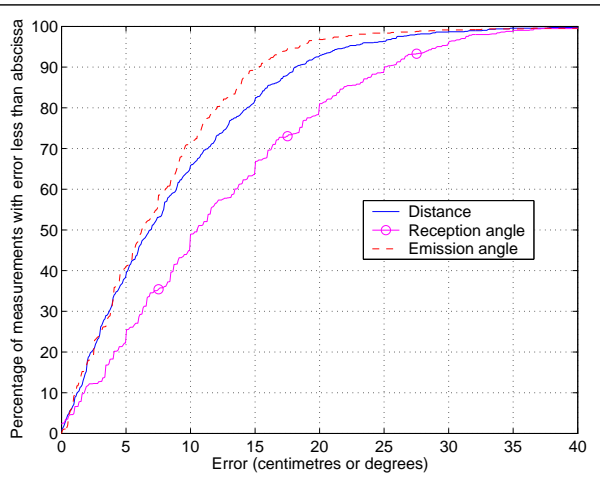

Figure 3. Raw measurement accuracy

As shown in figure 3 , the ninetieth percentile angular accuracies for reception and emission are $25^{\circ}$ and $15^{\circ}$, respectively. These deviate from the ideal limits stated in section 3 because the infrared transducers sensitivity beam is not perfectly aligned on the manually assembled prototypes 


\subsection{Location and orientation accuracy}

The measurement data for the experiments was fed into the map tracing and non-linear regression algorithms to produce relative location and orientation estimates. In order to compare the algorithms' results with the manuallymeasured locations and orientations, a coordinate space transform was calculated for each result. The coordinate space transform consists of the $2 \mathrm{D}$ rotation and translation which minimise the mean squared distance error between corresponding devices. The accuracy of the resulting locations and orientations are shown in figure 4 , and the ninetieth and ninety-fifth percentile accuracies are given in table 1 . Note that of the two algorithms, non-linear regression achieves superior accuracy, but both algorithms have a location accuracy better than that of the raw distance measurements provided by the devices.

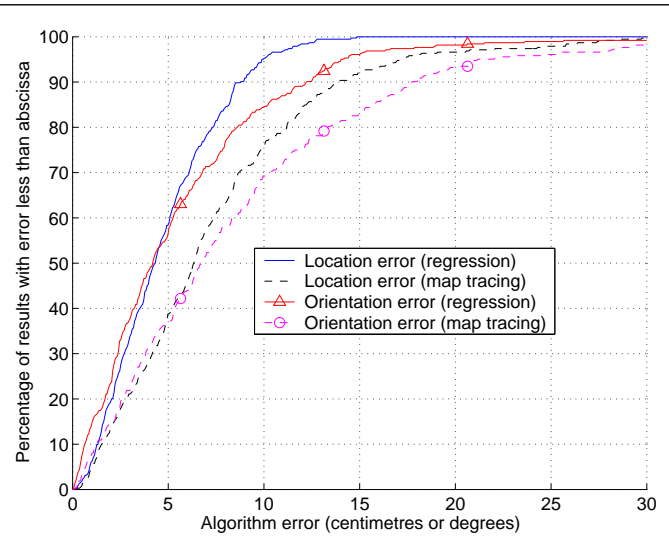

Figure 4. Algorithm accuracy distributions

\subsection{Evaluation}

Based on the findings with the prototype system, this section evaluates the use of infrared technology for finegrained positioning of mobile users' devices on surfaces.

Accuracy. The location and orientation accuracy of the system is largely suitable for the types of applications outlined in section 2.1, such as configuration of device connections, automatic interface export, and location-sensitive data sharing between meeting attendees.

Update rate and scalability. In the current hardware prototypes, each device's transmission sequence takes $600 \mathrm{~ms}$. With some modifications this can easily be reduced to less than $200 \mathrm{~ms}$ per device.

If line-of-sight between devices on the surface is minimal, then it may be necessary for all devices to transmit in order to uniquely solve for their locations and orientations.
Assuming a transmission duration of $200 \mathrm{~ms}$ and poor lineof-sight conditions on a surface with twenty objects, positioning updates would be available approximately every four seconds. Although this would be awkward for users desiring fast, automatic connection and data sharing, in our experiences with surface-based systems, line-of-sight conditions are rarely so poor, and positioning updates are often feasible after only three or four devices report their measurements. Although only four infrared devices were constructed, it has been experimentally verified that the wireless protocol employed by the devices' radio units will support twenty or more devices in simultaneous operation.

Environmental factors. The reflectance of the surface on which the devices are placed affects the received infrared light intensity. Although the devices perform similarly on typical desks and work surfaces, extremely shiny surfaces (such as glass) or absorbent surfaces (such as a black tablecloth) can cause additional ranging errors. Additionally, direct sunlight is crucial to the receivers used on the prototypes. section 2.1 , mobile users rarely choose surfaces which are in direct sunlight, since it makes device displays difficult to read. Also, a set of informal experiments have shown that e.g. additional automatic calibration can compensate for ranging errors on unusually reflective or absorbent surfaces.

Power Consumption. The prototypes have an average power consumption of $300 \mathrm{~mW}$ when actively generating measurements. The biggest part of this relatively high power consumption is used up by the infrared circuitry. Appropriate redesigns can easily reduce this amout to less than $50 \mathrm{~mW}$ by e.g. reducing the pulse length of the infrared light pulses.

\section{Conclusion}

In this paper, we have proposed a new type of positioning system for portable devices placed on surfaces. The system uses intensity measurements of infrared pulses transmitted between devices to determine their relative location and orientation. Unlike previous surface-based location techniques, the system is not reliant on any pre-installed infrastructure and thus provides a more practical solution for the surface-based computing tasks of mobile users. The prototypes (figure 1) were designed to fully gauge the potential of infrared for surface-based positioning. Clearly, they are too unwieldy and complex for everyday use as mobile devices. For a consumer electronic device, the number of transducers could be vastly reduced by choosing infrared emitters and detectors which have much wider sensitivity beams. We developed a lightweight map tracing algorithm and a more robust non-linear regression algorithm which estimate device locations and orientations using distance and angle measurements collected from the devices. The ac- 


\begin{tabular}{|c||c|c||c|c|}
\cline { 2 - 5 } \multicolumn{1}{c|}{} & \multicolumn{2}{c||}{ Ninetieth percentile } & \multicolumn{2}{c|}{ Ninety-fifth percentile } \\
\cline { 2 - 5 } \multicolumn{1}{c|}{} & Location & Orientation & Location & Orientation \\
\hline Raw values & $18 \mathrm{~cm}$ & $25^{\circ}$ & $22 \mathrm{~cm}$ & $28^{\circ}$ \\
Map tracing & $14 \mathrm{~cm}$ & $18^{\circ}$ & $17 \mathrm{~cm}$ & $21^{\circ}$ \\
Non-linear regression & $9 \mathrm{~cm}$ & $12^{\circ}$ & $10 \mathrm{~cm}$ & $14^{\circ}$ \\
\hline
\end{tabular}

Table 1. Accuracy summary for measurements and location algorithms

curacy and update rate of the prototype devices is appropriate for enhancing users' surface-based applications with location-awareness. Using the regression algorithm, ninetyfive percent of the positioning results returned by the system are accurate to $10 \mathrm{~cm}$ in location, and $14^{\circ}$ in orientation.

\section{References}

[1] James Scott, Frank Hoffmann, Glenford Mapp, Mike Addlesee, and Andy Hopper. Networked Surfaces: A new concept in mobile networking. ACM Mobile Networks and Applications, 7(5), October 2002.

[2] Jun Rekimoto and Masanori Saitoh. Augmented surfaces: A spatially continuous work space for hybrid computing environments. In Proceedings of the Conference on Human Factors in Computing Systems (CHI), pages 378-385, Pittsburgh, USA, May 1999.

[3] John Barton, Brad Johanson, Vikram Vijayaraghavan, Tony Hsieh, Tomoto Shimizu, and Armando Fox. The MeetingMachine: Interactive workspace support for nomadic users. In Proceedings of the Workshop on Mobile Computing Systems and Applications, pages 2-12, Monterey, USA, October 2003.

[4] Brygg Ullmer, Hiroshi Ishii, and Dylan Glas. mediaBlocks: Physical containers, transports, and controls for online media. In Proceedings of the Twenty-fifth International Conference on Computer Graphics and Interactive Techniques (SIGGRAPH), pages 379-386, Orlando, USA, July 1998. ACM.

[5] Mike Addlesee, Rupert Curwen, Steve Hodges, Joe Newman, Pete Steggles, Andy Ward, and Andy Hopper. Implementing a sentient computing system. IEEE Computer, 34(8):50-56, August 2001.

[6] Diego López de Ipiña, Paulo Mendonça, and Andy Hopper. TRIP: A low-cost vision-based location system for ubiquitous computing. Personal and Ubiquitous Computing, 6(3):206-219, May 2002.

[7] Brygg Ullmer and Hiroshi Ishii. The MetaDESK: Models and prototypes for tangible user interfaces. In Proceedings of the Symposium on User Interface Software and Technology (UIST), pages 223-232, Banff, Alberta, Canada, October 1997.

[8] John Underkoffler and Hiroshi Ishii. Urp: A luminoustangible workbench for urban planning and design. In Proceedings of the Conference on Human Factors in Computing Systems (CHI), pages 386-393, Pittsburgh, USA, May 1999.
[9] Frank Hoffmann and James Scott. Location of mobile devices using Networked Surfaces. In Proceedings of UbiComp: Ubiquitous Computing, pages 281-298, Göteborg, Sweden, September 2002.

[10] Albrecht Schmidt, Martin Strohbach, Kristof van Laerhoven, Adrian Friday, and Hans-Werner Gellersen. Context acquisition based on load sensing. In Proceedings of UbiComp: Ubiquitous Computing, pages 333-350, Göteborg, Sweden, September 2002.

[11] Ken Camarata, Ellen Yi-Luen Do, Brian D. Johnson, and Mark D. Gross. Navigational blocks: Navigating information space with tangible media. In Proceedings of the International Conference on Intelligent User Interfaces (IUI), pages 31-38, San Francisco, USA, January 2002.

[12] James Patten, Hiroshi Ishii, Jim Hines, and Gian Pangaro. Sensetable: a wireless object tracking platform for tangible user interfaces. In Proceedings of the Conference on Human Factors in Computing Systems (CHI), pages 253-260, Seattle, USA, April 2001.

[13] Roy Want, Andy Hopper, Veronica Falcao, and Jon Gibbons. The Active Badge location system. ACM Transactions on Information Systems, 10(1):91-102, January 1992.

[14] Roy Want, Bill Schilit, Norman Adams, Rich Gold, Karin Petersen, David Goldberg, John Ellis, and Mark Weiser. An overview of the ParcTab ubiquitous computing experiment. IEEE Personal Communications, 2(6):28-33, December 1995

[15] Dana Kirsch and Thad Starner. The Locust Swarm: An environmentally-powered, networkless location and messaging system. In Proceedings of the First International Symposium on Wearable Computers, Boston, USA, October 1997.

[16] Drexel Hallaway, Tobias Höllerer, and Steven Feiner. Coarse, inexpensive, infrared tracking for wearable computing. In Proceedings of the Seventh IEEE International Symposium on Wearable Computers (ISWC), pages 69-78, White Plains, NY, USA, October 2003.

[17] Greg Welch, Gary Bishop, Leandra Vicci, Stephen Brumback, Kurtis Keller, and D'nardo Colucci. The HiBall tracker: High-performance wide-area tracking for virtual and augmented environments. In Proceedings of the ACM Symposium on Virtual Reality Software and Technology, University College London, United Kingdom, December 1999.

[18] Andy Ward, Alan Jones, and Andy Hopper. A new location technique for the active office. IEEE Personal Communications, 4(5):42-47, October 1997. 